\title{
Antenatal diagnosis of intrauterine infection with coxsackievirus B3 associated with live birth
}

\author{
Annie Ouellet ${ }^{1}$, Rebecca Sherlock ${ }^{2}$, Baldwin Toye ${ }^{3}$ and Karen Fung Kee Fung ${ }^{1}$ \\ ${ }^{1}$ Division of Maternal-Fetal Medicine, Department of Obstetrics and Gynecology, University of Ottawa, \\ The Ottawa Hospital, Ottawa, Ontario, Canada \\ ${ }^{2}$ Division of Neonatology, Department of Pediatrics, The Children's Hospital of Eastern Ontario, Ottawa, \\ Ontario, Canada \\ ${ }^{3}$ Division of Microbiology, Department of Pathology and Laboratory Medicine, University of Ottawa, \\ The Ottawa Hospital, Ottawa, Ontario, Canada
}

Background: Prior reported cases of stillbirth and neonates infected with enteroviruses suggest transplacental infection. We present a case of fetal infection with coxsackievirus B3, diagnosed antenatally and resulting in live birth.

Case: A pregnant woman presented at 26 weeks with fetal tachycardia and non-immune hydrops fetalis. Coxsackievirus B3 was cultured from amniotic fluid. Maternal antibody to coxsackievirus B3 was positive at I: 512. At 32 weeks, the fetus deteriorated and was delivered. Cord blood antibody to coxsackievirus B3 was positive at a higher titer. Following neonatal death, brain and placental tissues were positive for enterovirus ribonucleic acid by polymerase chain reaction.

Conclusion: Intrauterine infection by enteroviruses should be considered in the differential diagnosis of nonimmune hydrops fetalis. Antenatal diagnosis of coxsackievirus B3 infection is associated with poor outcome.

Key words: EnTERovirus; Transplacental InFECTION; FetAl Hydrops

\section{PRÉCIS}

Intrauterine infection by enteroviruses is part of the differential diagnosis of non-immune hydrops fetalis, and is associated with poor prognosis.

\section{INTRODUCTION}

Enteroviruses, which include coxsackieviruses and echoviruses, are distributed worldwide and cause infections, which are more prevalent during the summer and early fall in temperate climates. Coxsackieviruses usually produce rela- tively mild symptoms in adults, although myocarditis and encephalitis are well recognized complications. Enterovirus infection of the human neonate is an unusual but serious event, which rivals neonatal herpes simplex virus infection in incidence and severity. Numerous reports of cases and epidemics can be found in the literature, documenting the morbidity and mortality associated with enteroviral infection in neonates ${ }^{1}$. However, transplacental infection and associated fetal morbidity and mortality have not been well documented. Detection of the virus has been reported in stillbirth infants at autopsy

Correspondence to: Annie Ouellet, MD, FRCSC, Division of Maternal-Fetal Medicine, Department of Obstetrics and Gynecology, Room 8420, The Ottawa Hospital, General Campus, 501 Smyth Road, Ottawa, ON K1H 8L6, Canada.

Email: aouellet@ottawahospital.on.ca 
and, most recently, in the placental tissues of newborns with neurodevelopmental delays ${ }^{2}$.

Antenatal enterovirus infection due to coxsackievirus B1 and echovirus types 11 and 27, has previously been isolated from amniotic fluid in the third trimester of pregnancies, with poor early neonatal outcome, supporting probable transplacental passage. Coxsackievirus B3 has been diagnosed in a stillborn infant with myocarditis and hydrops fetalis ${ }^{3}$. Post-natal diagnosis of coxsackievirus B3 has been made from congenital skin lesions ${ }^{4}$. We present a case of intrauterine infection by coxsackievirus B3 diagnosed antenatally, resulting in live birth.

\section{CASE REPORT}

At a regular prenatal visit, a 34-year-old Caucasian presented at 26 weeks gestation with fetal tachycardia of 175 beats per minute. The pregnancy had otherwise been uneventful. Two previous ultrasounds, at 7 and 19 weeks, were reported as normal. There was no history of febrile illnesses during the pregnancy. The patient had previously delivered a normal term baby, vaginally. Her past medical and surgical history was unremarkable. Her blood group was $\mathrm{O}$ positive with a negative antibody screen. The patient was not taking any medication, did not smoke, drink alcohol or use illicit drugs.

An ultrasound showed a fetal hydrops with significant ascites and a small pericardial effusion. Mild scalp and thoracic skin edema were present without pleural effusion. No structural fetal anomalies were noted. The fetal biophysical profile was $8 / 8$. The umbilical artery Doppler was normal, as was the middle cerebral artery peak systolic velocity.

Fetal echocardiography showed no obvious structural cardiac abnormalities. A fetal heart rate (FHR) of 165-175 beats per minute with 1: 1 atrioventricular conduction was confirmed. Frequent premature atrial contractions with several brief runs of supraventricular tachycardia (rate 245-260 beats per minute) were observed. Decreased ventricular function (ejection fraction $20-25 \%$ ) with small pericardial effusion was noted. A bright-walled atrium without evidence of ventricular endocardial fibroelastosis was viewed.
Diagnostic amniocentesis was performed. The fetal karyotype was $46 \mathrm{XY}$. Serological testing for toxoplasma, rubella, parvovirus B19, cytomegalovirus, influenza and Epstein-Barr virus did not indicate an acute infection. HIV serology was also negative. Maternal therapy with Digoxin 500 mcg BID and Sotalol $80 \mathrm{mg}$ BID was started to control the tachyarrythmia. Betamethasone was administrated prophylactically to accelerate fetal lung maturity.

Following a week of antiarrythmic therapy within the therapeutic range, the fetal status remained unchanged. A diagnostic cordocentesis demonstrated a fetal hemoglobin at $11 \mathrm{gm} / \mathrm{dL}$, hematocrit 32\%, MCV $133.7 \mathrm{fL}$ and mild thrombocytopenia $99,000 / \mathrm{mm}^{3}$. The fetal hepatic and renal function tests were normal.

The amniotic fluid was subsequently reported as culture positive for an enterovirus, although it was negative for enterovirus RNA by nucleic acid sequence-based amplification (NASBA; bioMérieux Inc.), and negative for parvovirus B19 DNA by polymerase chain reaction (PCR). The enterovirus isolate was sent to the National Centre for Enteroviruses (NCEV) in Halifax, Canada and was typed as a coxsackievirus type B, group 3 (B3). Maternal cultures of stool and throat washings were negative for enteroviruses. Maternal serum obtained at initial presentation contained neutralizing antibody to coxsackievirus B3 at a titer of 1: 512 (NCEV). A repeat serum taken 3 weeks later remained positive for antibody to coxsackievirus B3 at the same titer.

The mother was kept on Sotalol, and the fetal heart rate normalized at 155 beats per minute, with minimal variability on the fetal heart monitoring. The fetal hydropic status remained unchanged. The infection status, the suspected poor fetal prognosis and the limited therapeutic options were discussed with the parents.

At 32 weeks gestation, the fetus developed oligohydramnios, bilateral pleural effusion, and worsening of the abdominal ascitis with a low biophysical profile score of $4 / 8$. A male infant, in breech presentation, was delivered with Apgar scores of four, six and eight at 1, 5 and 10 minutes respectively. The birth weight was $2380 \mathrm{~g}$. Umbilical artery cord gas showed pH: 7.33; $\mathrm{PO}_{2}$ 42; $\mathrm{PCO}_{2} 19.8$ and $\mathrm{HCO}_{3}$ 21.9. Anasarca was 
present. There were no spontaneous respirations and the initial heart rate was less than $80 \mathrm{bpm}$. The infant was resuscitated with positive pressure ventilation and intubation. Initial physical exam revealed a grossly edematous male infant with marked central edema. There were no dysmorphic features or obvious congenital anomalies.

Paracentesis yielded $30 \mathrm{ml}$, and thoracentesis produced $11 \mathrm{ml}$ of sero-sanguinous fluid. The initial hemoglobin was $11 \mathrm{gm} / \mathrm{dL}$, platelets were $65,000 / \mathrm{mm}^{3}$ and white cell count was $4.2 / \mathrm{mm}^{3}$. Coagulation studies revealed at partial tromboplastine time of 46 and an international normalization ratio of 1.46. Packed red blood cells, platelets and fresh frozen plasma were given. There were no arrhythmias recorded at or around the time of delivery. Dopamine was initiated to enhance renal perfusion. The infant remained ventilator-dependent with high oxygen needs. An early echocardiogram revealed significant systolic dysfunction with a shortening fraction of $25 \%$. It also revealed severe diastolic dysfunction with stiff atria and ventricles. There was evidence of hepatic dysfunction with elevated coagulation studies and decreased albumin, despite fresh frozen plasma, vitamin $\mathrm{K}$ and albumin infusions. The infant became anuric after the first 24 hours of life.

PCR for Herpes group viruses and enteroviruses and viral cultures of the infant's blood, pleural, ascitic, and cerebrospinal fluids were negative. Cord blood obtained at birth demonstrated a neutralizing antibody titer to coxsackievirus B3 of 1: 16384 (NCEV). With prenatal detection of the specific viral pathogen, the infant was treated with Pleconaril as an emergency release medication as this antiviral drug has not been approved in Canada. With evidence of multiorgan failure, on the fourth day of life, therapy was withdrawn and the baby expired.

The autopsy revealed a hydropic baby without gross anomalies. Cardiac pathology included a mild right ventricular hypertrophy, biventricular dilatation and two small foci of calcifications (right ventricule and atrioventricular groove). The neuropathological evaluation did not reveal any evidence of viral involvement. The placenta was hydropic and displayed dysmature villi. There was mild microscopic meconium staining of the membranes, small areas of infarcts and fibrin, but no evidence of any acute or chronic placental inflammation. Although viral cultures were all negative, PCR (Chemicon International) for enterovirus RNA was positive from brain and placenta tissues, and negative for the other tissues (lung, spleen, liver, kidney, and heart).

\section{DISCUSSION}

Despite reports of intrauterine transmission of enteroviruses in animals ${ }^{5}$, vertical transmission in humans is not well accepted. This report presents a case of intrauterine coxsackievirus B3 infection diagnosed during the second trimester with nonimmune fetal hydrops, resulting in a live birth and subsequent early neonatal death, secondary to multiorgan failure. A case of coxsackievirus B3 reported in $1970^{6}$ describing a stillborn hydropic fetus appears similar to our case, supporting the transplacental passage and the poor outcomes associated with this type of intrauterine infection. Other cases of stillbirth with post-natally diagnosed enteroviral infection have been reported in the literature ${ }^{7}$ Euscher $e t$ al. recently reported cases of placental infection with coxsackievirus, possibly resulting in encephalitis with subsequent neurological damage in the newborn ${ }^{2}$. We were able to detect the presence of enteroviral RNA in both brain and placental tissue, supporting the findings of that study. Our case also demonstrates that transplacental infection by enteroviruses in humans does occur, as does the potential for diagnosing it prenatally. Coxsackieviruses and other enteroviruses can cause myocarditis, and therefore, intrauterine infection with enteroviruses should be considered in the differential diagnosis of non-immune hydrops fetalis.

In adults most infections due to enteroviruses are asymptomatic, as was seen in our case. A nonspecific febrile illness with or without a rash may occasionally be present. Other less commonly encountered clinical manifestations of enteroviral infections include encephalitis, aseptic meningitis, pericarditis and myocarditis. The antenatal diagnosis was made by a positive culture of the amniotic fluid, although there was a false-negative NASBA result. Nucleic acid amplification tests (NAAT) such as PCR and NASBA are now 
being used more frequently in support of virologic diagnosis, but there are some limitations. Despite the reported enhanced sensitivity of many of these new molecular assays over enterovirus culture, most of the published evaluations have involved the testing of cerebrospinal fluid rather than amniotic fluid ${ }^{6}$. The viral RNA may degenerate more easily in amniotic fluid or there may be inhibitors present. In our case, the culture of the amniotic fluid was performed in addition to NASBA. This led to maternal diagnosis, which was supported by the positive serology results. Infection of the infant was suspected by the higher titers of antibodies found in the cord blood (we could not exclude that the cord antibodies could be of maternal origin), and confirmed by the presence of enteroviral RNA in brain and placenta tissues at autopsy. Nucleic acid amplification methods such as PCR have a lower limit of detection than viral cultures, which would explain why the autopsy tissues were PCR positive but culture negative. Also, as previously reported, viral infections of the placenta are associated with several non-specific histologic findings ranging from focal to diffuse. Such viruses may require extensive sampling to be detected, which accounts for the non-specific histologic results on the placenta in our case.
Prenatal diagnosis of intrauterine infection leads to increased opportunity for fetal therapy. The empiric treatments proposed for neonates infected with enteroviruses have included combined therapy of intravenous gamma or hyperimmune globulin and leukocyte interferon ${ }^{8}$. This has not been reported for in utero therapy, and there is no evidence that such treatments will prevent the fatal outcome of disease. Specific antiviral therapy such as Pleconaril shows promise in the treatment of meningitis and other life threatening infections due to enteroviruses. However, the safety or efficacy of this drug during pregnancy is unknown. Given the poor fetal prognosis, it would seem appropriate to discuss this antiviral treatment option with the parents. The results of on-going studies of Pleconaril may determine what role, if any, this antiviral drug has in the treatment of antenatal and perinatal enteroviral infections ${ }^{9}$.

\section{ACKNOWLEDGEMENTS}

The authors would like to thank Dr Spencer Lee and his staff at the National Centre for Enteroviruses in Halifax for the typing of the coxsackievirus and the serological testing.

\section{REFERENCES}

1. Modlin JF. Perinatal echovirus and group B coxsackievirus infections. Clin Perinatol 1988; 15:233-46

2. Euscher E, Davis J, Holzman I, et al. Coxsackievirus infection of the placenta associated with neurodevelopmental delays in the newborn. Obstet Gynecol 2001;98:1019

3. Bates HR Jr. Coxsackievirus B3 calcific pancarditis and hydrops fetalis. Am J Obstet Gynecol 1970; 106(4):629-30

4. Sauerbrei A, Gluck B, Jung K, et al. Congenital skin lesions caused by intrauterine infection with coxackievirus B3. Infection 2000;28(5):326-8

5. Modlin JF, Bowman M. Perinatal transmission of coxsackievirus B3 in mice. J Inf Dis 1987;156:21 - 5
6. Smalling TW, Sefers SE, Li H, et al. Molecular approaches to detecting herpes simplex virus and enteroviruses in the central nervous system. J Clin Microbiol 2002;40:2317-22

7. Brady WK, Purdon A. Intrauterine fetal demise associated with enterovirus infection. South Med J 1986;79:770-2

8. Abzug MJ, Keyserling HL, Lee ML, et al. Neonatal enterovirus infection: virology, serology, and effects of intravenous immune globulin. Clin Infect Dis 1995;20:1201-6

9. Rotbart HA, Webster AD, for the Pleconaril Treatment Registry Group. Treatment of potentially life-threatening enterovirus infections with Pleconaril. Clin Infect Dis 2001;32:228-35 


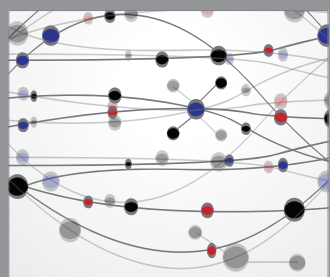

The Scientific World Journal
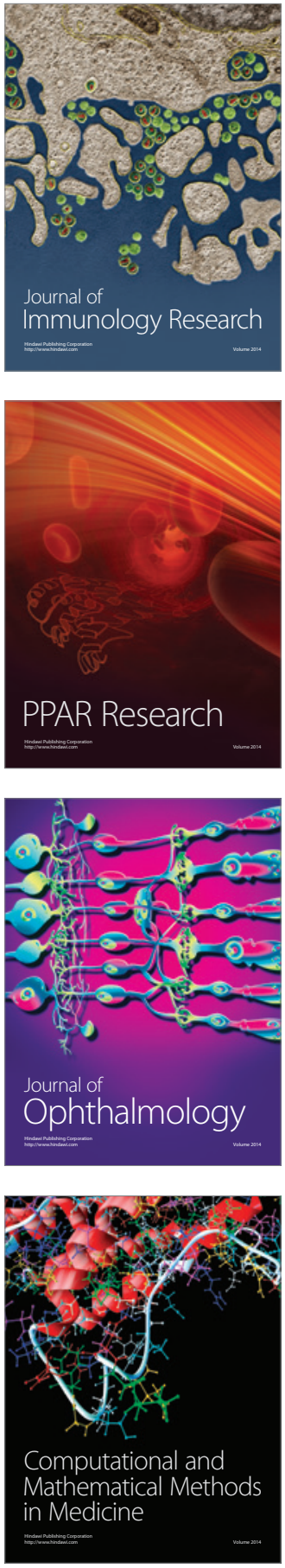

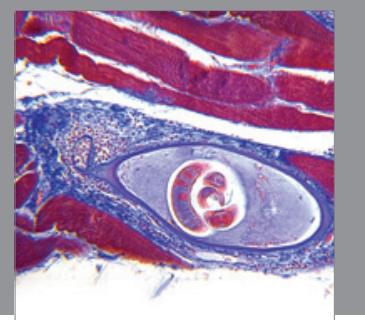

Gastroenterology

Research and Practice
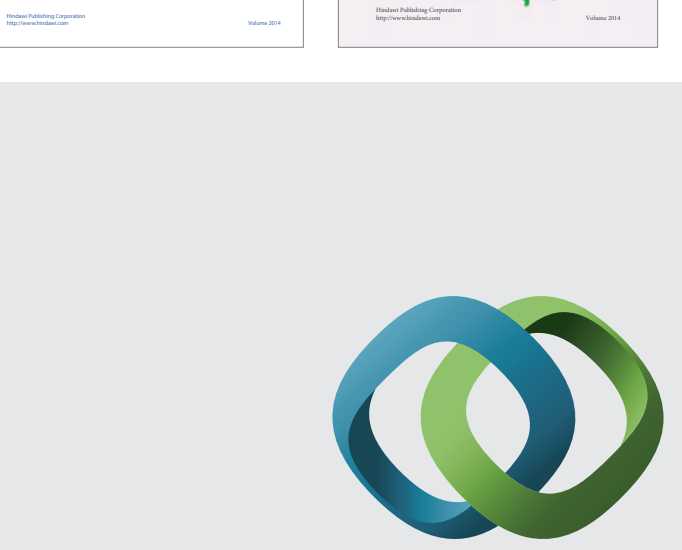

\section{Hindawi}

Submit your manuscripts at

http://www.hindawi.com
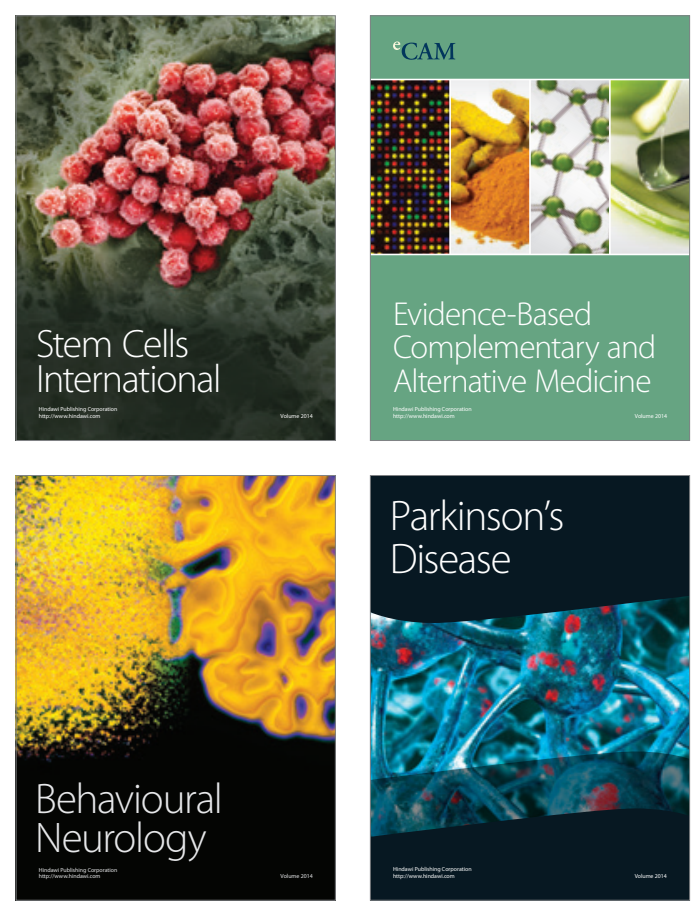

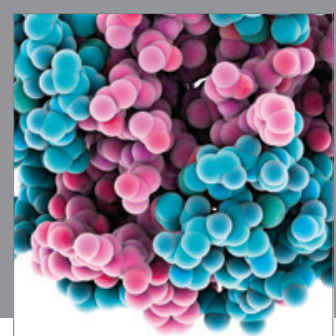

Journal of
Diabetes Research

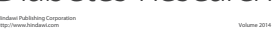

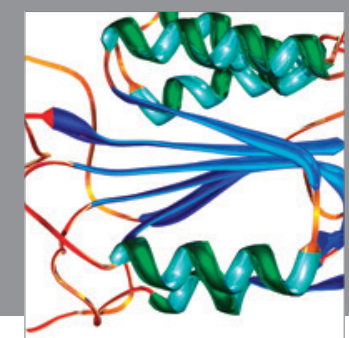

Disease Markers
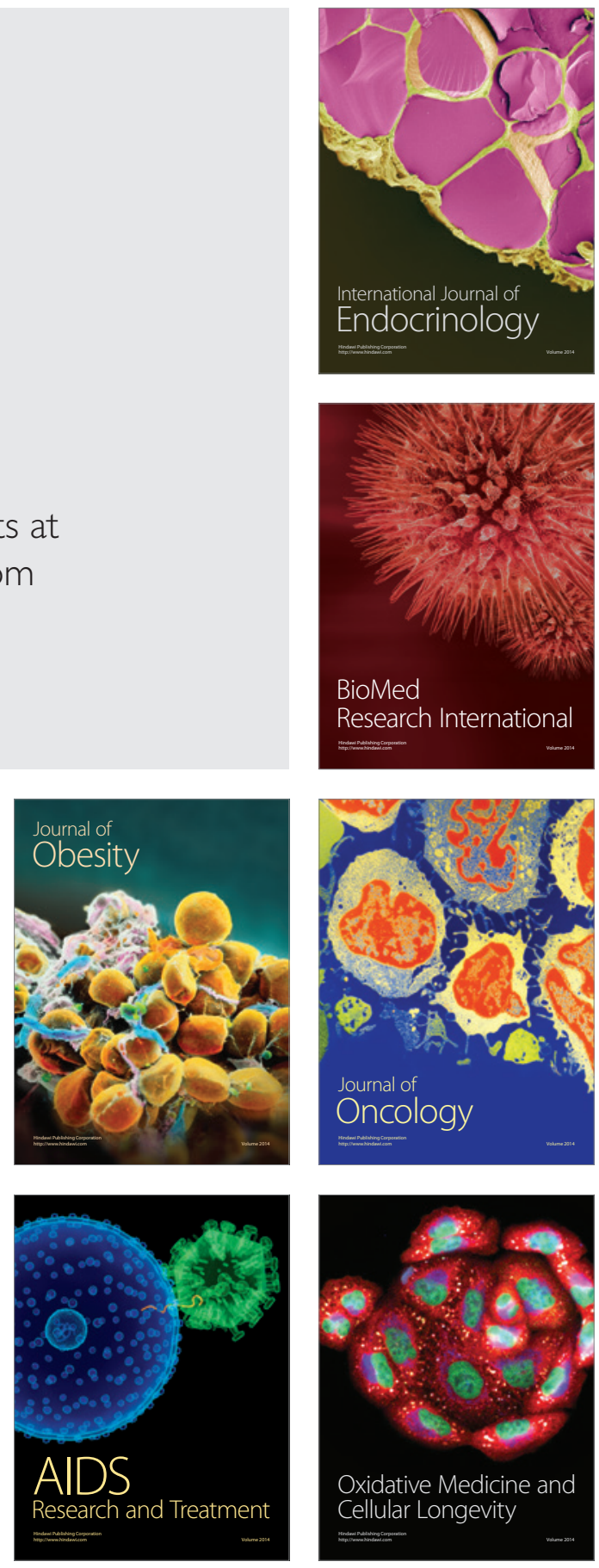